\title{
Some Thoughts on Assessing near Bed Surface Flow Hydrodynamics using Instrumented Particles
}

\author{
Khaldoon ALOBAIDI and Manousos VALYRAKIS ${ }^{\varpi}$ \\ Water Engineering Lab, School of Engineering, University of Glasgow, Glasgow, UK \\ Manousos.Valyrakis@glasgow.ac.uk
}

\begin{abstract}
A novel and low cost tool, namely the instrumented particle, that is potentially able to directly monitor the near bed surface flow hydrodynamics and can be used by researchers and practitioners alike is presented. The particle is fitted with micro-electro-mechanical-systems, MEMS, sensors that are able to quantify its inertial dynamics and detect the particle's incipient motion accurately. A well-controlled laboratory flume experiment for assessing the incipient entrainment of the instrumented particle which mimics the behaviour of a naturally rounded pebble resting on a riverbed for a range of flowrates near the threshold of motion is conducted. The logged acceleration readings, after appropriate post-processing, are used to assess the entrainement threshold of the instrumented particle. Appropriate theories that take into account the dynamic characteristics of particle's entrainment are considered to interpret these readings with an ultimate goal of back-estimating hydrodynamic drag which is representative of the hydrodynamic forces acting on the bed-surface.
\end{abstract}

Keywords: instrumented particle, impulses, MEMS sensors, incipient motion, turbulence.

\section{INTRODUCTION}

Sediment entrainment is considered to be the governing process in different applications around the fields of geoscience and engineering and therefore has gained a lot of attention in the literature for the past century (Buffington and Montgomery 1997). Therefore, extensive field and laboratory studies exist in the literature for assessing the conditions that can result in initiation of sediment entrainment, namely incipient motion. Different criteria for assessing the incipient entrainment of sediment particles have been suggested by different authors (Shields 1936; Bagnold 1966; Valyrakis et al. 2010). Among the different criteria, the impulse (or energy) criterion 
is the only criterion that considers both the force magnitude of the near bed turbulent event and it duration are relevant in predicting sediment entrainment. The field and laboratory studies of sediment entrainment are usually performed using expensive tools like Acoustic Doppler Velocimetry (ADV) and particle image velocimetry (PIV). The recent technological advancement has provided researchers in the field of fluvial hydraulics with the ability to directly assess sediment entrainment using low cost tools like micro-electro-mechanical-systems, MEMS, sensors (Valyrakis and Pavlovskis 2014; Valyrakis and Alexakis 2016; Al-Obaidi et al. 2020; AlObaidi and Valyrakis 2021). Researchers and practitioners alike can benefit from using such low cost sensors in comparison to the expensive velocimetry techniques that indirectly offer estimates of shear stresses that could be linked to sediment entrainment via Shields diagram. Hence, the motivation of this work is to study the application of instrumented particles as low cost tools for assessing near-bed flow hydrodynamics with an ultimate goal of back-estimating hydrodynamic drag which is representative of the hydrodynamic forces acting on the bed-surface.

\section{THE INSTRUMENTED PARTICLE}

The instrumented particle used in this study is $7 \mathrm{~cm}$ in diameter that is embedded with microelectromechanical (MEMS) sensors (a tri-axial accelerometer, a tri-axial gyroscope, and a magnetometer) that record at the same frequency which is adjustable with a range of $100-500 \mathrm{~Hz}$. Depending on the flow conditions and expected particle's response, one can define a sufficient logging frequency (for this study a logging frequency of $150 \mathrm{~Hz}$ is selected). The sensors are interconnected forming inertial measurement units (IMUs) with which the particle's inertial dynamics can be precisely quantified.

\section{EXPERIMENTS AND RESULTS}

A water recirculating flume that is $8 \mathrm{~m}$ in length, $0.9 \mathrm{~m}$ in width and is able to carry flows of up to $0.4 \mathrm{~m}$ deep is used for the study. The particle is placed on micro-topography made of 4 hemispheres forming a triangular arrangement in the test section that is located $2.5 \mathrm{~m}$ downstream the outlet and $5.5 \mathrm{~m}$ upstream the inlet of the flume. The location of the test section ensures having hydraulically rough fully developed turbulent flow. For a range of flowrate that represents the for near-critical flow conditions, six run are performed to investigate the incipient entrainment of the instrumented particle. A video camera is placed on one side of the flume near the test section in order to record the particle's movements accurately. The logged readings of the sensors (20 minutes for each run) are post-processed using inertial sensor fusion (Kalman filtering) to reduce the uncertainty (by using the readings from one sensor to correct the readings

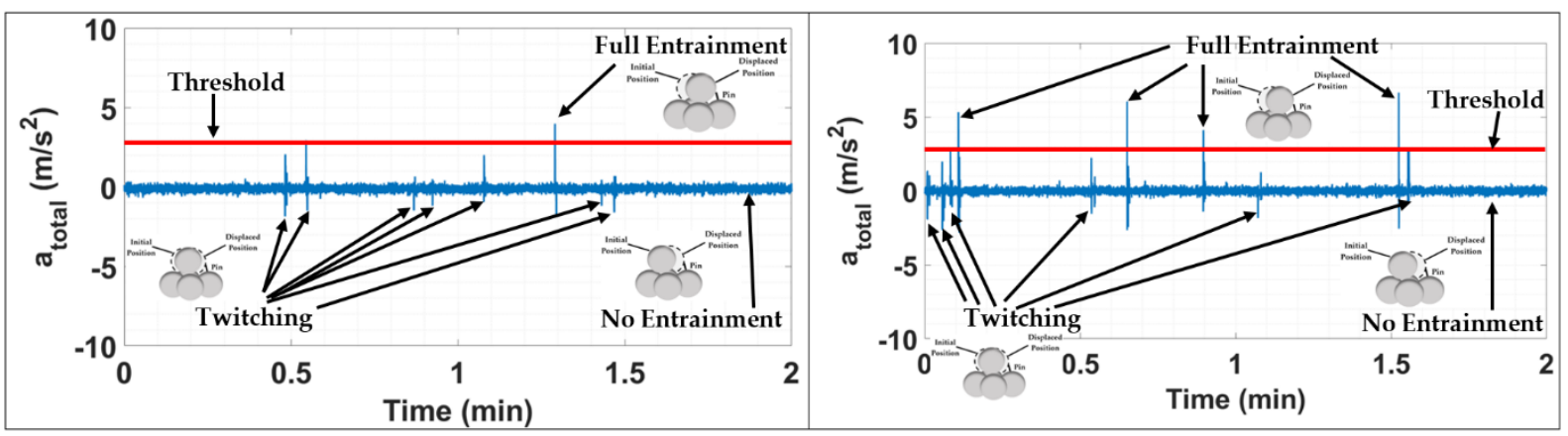

Fig. 1. The corrected total acceleration (estimated by inertial sensor fusion of the logged readings of the instrumented particle) for two runs of the experiment with the red line showing the threshold separating the full entrainments from the twitching events (or the partial entrainments) as validated by the visual assessment. 
of the other sensor) and the corrected acceleration readings are used to estimate the total acceleration of the instrumented particle (samples of two minutes are shown in Fig. 1). The total acceleration results are then used to assess the entrainment of the instrumented particle for the six runs of the experiment by defining a threshold value separating the full entrainments from the twitching events (or the partial entrainments) that is validated by manual comparison to the video recordings. Additionally, the acceleration readings are linked to the velocities of the turbulent flow events via relevant theories of flow impulses (Valyrakis et al. 2010).

\section{References}

Al-Obaidi, K., and M. Valyrakis (2021), A sensory instrumented particle for environmental monitoring applications: development and calibration, IEEE Sens. J. 21, 8, 10153-10166, DOI: 10.1109/ JSEN.2021.3056041.

Al-Obaidi, K., Y. Xu, and M. Valyrakis (2020), The design and calibration of instrumented particles for assessing water infrastructure hazards, J. Sens. Actuator Netw. 9, 3, 36, DOI: 10.3390/jsan 9030036.

Bagnold, R.A. (1966). An Approach to the Sediment Transport Problem from General Physics, Geophysical Survey Professional Paper 422-I, US Government Printing Office, Washington.

Buffington, J.M., and, D.R. Montgomery (1997), A systematic analysis of eight decades of incipient motion studies, with special reference to gravel-bedded rivers, Water Resour. Res. 33, 8, 19932029, DOI: 10.1029/96WR03190.

Shields, A. (1936). Anwendung der Aehnlichkeitsmechanik und der Turbulenzforschung auf die Geschiebebewegung, Ph.D. Thesis, Technical University Berlin.

Valyrakis M., and A. Alexakis (2016), Development of a "smart-pebble" for tracking sediment transport, In: Proc. 8th Int. Conf. Fluvial Hydraulics (River Flow 2016), St. Louis, USA.

Valyrakis, M., and E. Pavlovskis (2014), "Smart pebble" design for environmental monitoring applications. In: Proc. 11 th. Int. Conf. Hydroinformatics (HIC 2014), New York City, USA, 2684-2687.

Valyrakis, M., P. Diplas, C.L. Dancey, K. Greer, and A.O. Celik (2010), Role of instantaneous force magnitude and duration on particle entrainment, J. Geophys. Res. Earth. Surf. 115, F02006, DOI: $10.1029 / 2008 J F 001247$. 